\title{
Evaluación del Estado Nutricional en Lactantes y Preescolares Atendidos en Centros de Recuperación Nutricional
}

\author{
Dr. Fernando Vio del R.; Dra. Cecilís Albala B.; Nutr. Sonia Olivares C.; \\ Nutr. Margarita Andrade S.1. \\ Nutritional Changes in Infants and Children \\ Attending Day Care Centers for the Undernourished
}

\begin{abstract}
As patt of a project on "A community integrated action to prevent and treat undernutrtion", lour day care centers were established in Southern Sintiago. The program was aimed to infants and preschool children who ware undernourished or at nutritional risk and was desgned to provide both 8 hours $\mathrm{x}$ day psychomotor stimulation and $1.200 \mathrm{Keal} x$ day diet. An evaluation carried out on 70 children showed that they were being served $985 \mathbf{k c a l} x$ day, and that their actual average food intake was $60 \%$ of the servings. Nutritional status based on weight for age rOMS standard) slowed that $76 \%$ of the children were undernourished. Height for age assesment showed that $59 \%$ were below the standard. Six months later, after corrective measures were tuken, the dict energy content had increased to $1.123 \mathrm{kcal} x$ day and actual food inlake to $75 \%$ of this. Weight for age improved by $17 \%$ ip $<0.001)$ and height for age by $30 \%$ ( $p<0.0005$ ). This nutritional recovery shows that careful nutritional cvaluation of day carc centers is essential if the goals are to be tulfilled.

(Key words: Caloric intake. Day care centers. Nutritional Clanges. Undernutrition).
\end{abstract}

La Desnutrición o Enfermedad for Déficit Nutricional, continúa siendo un problema importante de Salud Pública en el país, aún cuando en los últimos 20 años se ha producido una mejoría apreciable en los indicadores de Salud y Nutrición a pesar del deterioro economico general y en especial de los estratos de menor ingreso ${ }^{1}$

Nadie duda de la relación directa que existe entre el nivel de vida de una población determinada y su estado nutricional. Sin embargo, en Chile esta relación no se dá en forma tan mecánica por la existencia de programas de intervención en Salud, especialmente el Programa Nacional de Alimentación Complementaria (PNAC) ${ }^{2}$. En Chile, la prevalencia de Desnutrición ha ido dimminuyendo, en niños menores de 6 años bajo control en establecimientos del Ministerio de Salud, evaluados por peso parat edad según curva patrón de SEMPE. de una prevalencia de $15.5 \%$ en 1975 , hasta un $8.8 \%$ en $1982^{3}$. En el Servicio de Salud Metropolitano Sur Oriente, con 600.000 habitantes y un $27.4 \%$ de extrema pobreza, la población de niños bajo control era de 45.000 en 1978, con una prevalencia de desnutrición de un 19.4\%. En 1983. la población de niños bajo control era de

1. Instituto de Nutrición y Tecnología de los Atimentos (INT A), Universidad de Chile.
60.000 con una prevalencia de Desnutricion de un $11 \%$. Esta importante disminución se debió en gran medida a que este servicio cuenta con un desarrollo razonabic de la atención primarìa con una cobertura del $85 \%$ de la población beneficiaria estimada $y$ a un programa de alimentación complementaria adecuado, habiendo sido el primer Servicio de Salud que en 1976, con un año de anticipación a la norma nacional. implantó la obligatoriedad del control de salud del niño sano y esquema completo de vacunación para la entrega de leche y alimentos proteicos del programa de alimentación complementaria ${ }^{2}$. Sin embargo, $y$ por existir un sector importante de extrema pobreza, se decidió establecer un proyecto de investigación en la acción en una de las comunas del Servicio de Salud Sur Oriente tendiente a enfrentar el problema de la Desnutrición. Así fue como entre el Instituto de Nutrición y Tecnologia de los Alimentos ([NTA), la Municipalidad de La Florida y la Inter American Foundation ( $A \mathrm{IF}$ ) se elaboró en 1981 el proyecto "Una acción integral para la prevención y tratamiento de la Desnutrición Infantil", que en una primera etapa de diagnóstico durante 1982 pudo establecer que en la comuna de La Florida existían campanentos y poblaciones de extrema pobreza con una mayor prevalencia de Desnutrición ${ }^{4}$. Para enfrentar este problema, se acordó en 1983, la creación 
de 4 Centros Comunitarios Educativo Nutricionales ubicados en los lugares de más fácil acceso para los niños desnutridos o en riesgo de desnutrir, $y$ atendidos por personal de la propia comunidad.

El objetivo de este trabajo es presentar la evaluación de la intervención nutricional efectua da a los niños asistentes a los Centros en un período de 6 meses.

\section{MATERIAL Y METODOS}

El programa de intervención nutricional de 210 niños desnutridos leves o en riesgo de desnutrir asistentes a los Centros, consistió en una atención de 8 horas diarias con estimulación psicomotora, supervisado por personal de la comunidad entrenado para tal efecto, y una dieta de $1.200 \mathrm{Kcal}$. por día distribuida en 3 comidas, lo que correspondería aproximadamente a un $60 \%$ de los requerimientos tutricionales, según FAO/OMS $73^{5}$. Además, y en forma paralela, se efectuó un programa educativo en salud y nutrición para los Directores y personal de los Centros, y para los padres de los niños ${ }^{6}$. La Evaluación consistió en medir la dieta que efectivamente recibian los niños, para lo cual se tomaron muestras de las bandejas con comida cntregadas a los niños y su posterior análisis químico-proximal y de ingredientes en el Laboratorio de la División de Tecnología de los Alimentos del INTA. Además se efectuó una encuesta por pesada de los reziduos efectuada por nutricionistas para medir ingesta real. Esta evaluación de dieta e ingesta real se efectuó en Mayo y Octubre de 1984. El análisis químico-proximal de Octubre de 1984 se efectuó en el Laboratorio de Ingenjería Química del Departamento de Investigaciones Cient ificas y Tecnológicas de la Pontificia Universiđad Católica de Chile.

La medicion del estado nutricional de los niños la efectú́ mensualmente, primero el equipo de salud del Consultorio durante 3 meses (Mayo-Junio y Julio) y luego continuó la Nutricionisti del Proyecto (Agosto 1984 y en adelante). Los niños eran pesados y medidos en balanzas y pedómetros calibrados. La información era registrada en formularios especiales que eran entregades todos los meses al equipo de salud y nutrición del INTA para su análisis. Para ésto se utjlizaron las curvas patrón SEMPE ${ }^{7}$ y $\mathrm{OMS}^{8}$ con las relaciones Peso/Edad, Talla/Edad y Peso/ Talla, sepatados en 2 grupos; lactantes y preescolares. Del total de niños asistentes a los Centros se tomó una muestra de 70 niños ( 15 lactantes y 55 preescolares), correspondientes a un $30 \%$ de los niños asistentes, los cuales tenian buena asistencia y contaban con información de peso y talla en los 6 meses de observación (Mayo a Octubre 1984).

\section{RESULTADOS}

En Mayo se estudiaron los 4 Centros, encontrándose que la dieta entregada a los nif̧os era tanto para lactantes como preescolares, de un promedic de $985 \mathrm{Kcal} / \mathrm{d}$ ía, en ve7 de las 1.200 $\mathrm{Kcal} / \mathrm{dia}$ ofrecidas. Al analizar la ingesta real en los Centros, el promedio para lactantes era de $521 \mathrm{Kcal} / \mathrm{dia}$, es decir, un $53 \%$ de lo servido, y para preescolares de $708 \mathrm{Kcal} / \mathrm{d}$ ía $(72 \%$ de lo entregado). Despues de 6 meses de intervención. y habiéndose adoptado severas medidas de control de preparación de la dieta y de la ingesta por parte del personal de los Centros, supervisados por nutricionistas especialmente contraladas. se encontró en Octubre 1984 que la dieta servida a los niños tenja como promedio en los 4 Centros $1.123 \mathrm{Kcal} / \mathrm{d}$ ia. La ingesta promedio para lactantes habia aumentado i $667 \mathrm{Kcal}$ idía $(60 \%$ de 10 entregado) y para preescolares a $890 \mathrm{Kcal} / \mathrm{d}$ ia ( $80 \%$ de lo servido).

El estado nutricional en Mayo de 1984 para lis 70 niños demostraba. para peso/edad. un $55.7 \%$ de Desnutrición según SEMPÉ y un $76 \%$ según OMS. Para tallaiedad, el déficit según SEMPE era de un $38.6 \%$ y de un $58.6 \%$ por OMS. Con peso/talla la desnutrición según SEMPE era sólo de un $15.7 \%$ y con OMS $17 \%$. Al separarlo según grupos de edad, en los lactantes la desnutrición era superior a la de los preescolares.

Después de 6 meses de intervención nutricional, asistencial y educativa en estos 70 niños, se apreciaron los siguientes resultados: la desnutrición peso/edad SEMPE había bajado a un $35.7 \%$ $y$ con OMS a un $59 \%$ siendo en ambos casos una disminución significativa $(p<0,001$ y $p<0,014$ respectivamente). Para talla/edad SEMPE, la dismimución fue a un $20 \%(\mathrm{p}<0.0005)$ y para OMS llegó a un $28.6 \%$ ( $p<0.0005$ ). Con peso/talla no hubo variación significativa (Figura 1). En los dos casos en que hubo disminución de los indicadores de Desnutrición (peso/edad y talla/edad) esta mejoría fue muy superior para los lactantes que para los preescolares.

\section{DISCUSION}

Siendo la Desnutrición todavia un problema importante de Salud Pública en el pais, que según Puffer y Serrano ${ }^{9}$ había explicado como causa básica o asociada el $57 \%$ de las defunciones de menores de 5 años acaecidas a fines de los años 60 en la ciudad de Santiago y comunas rurales 


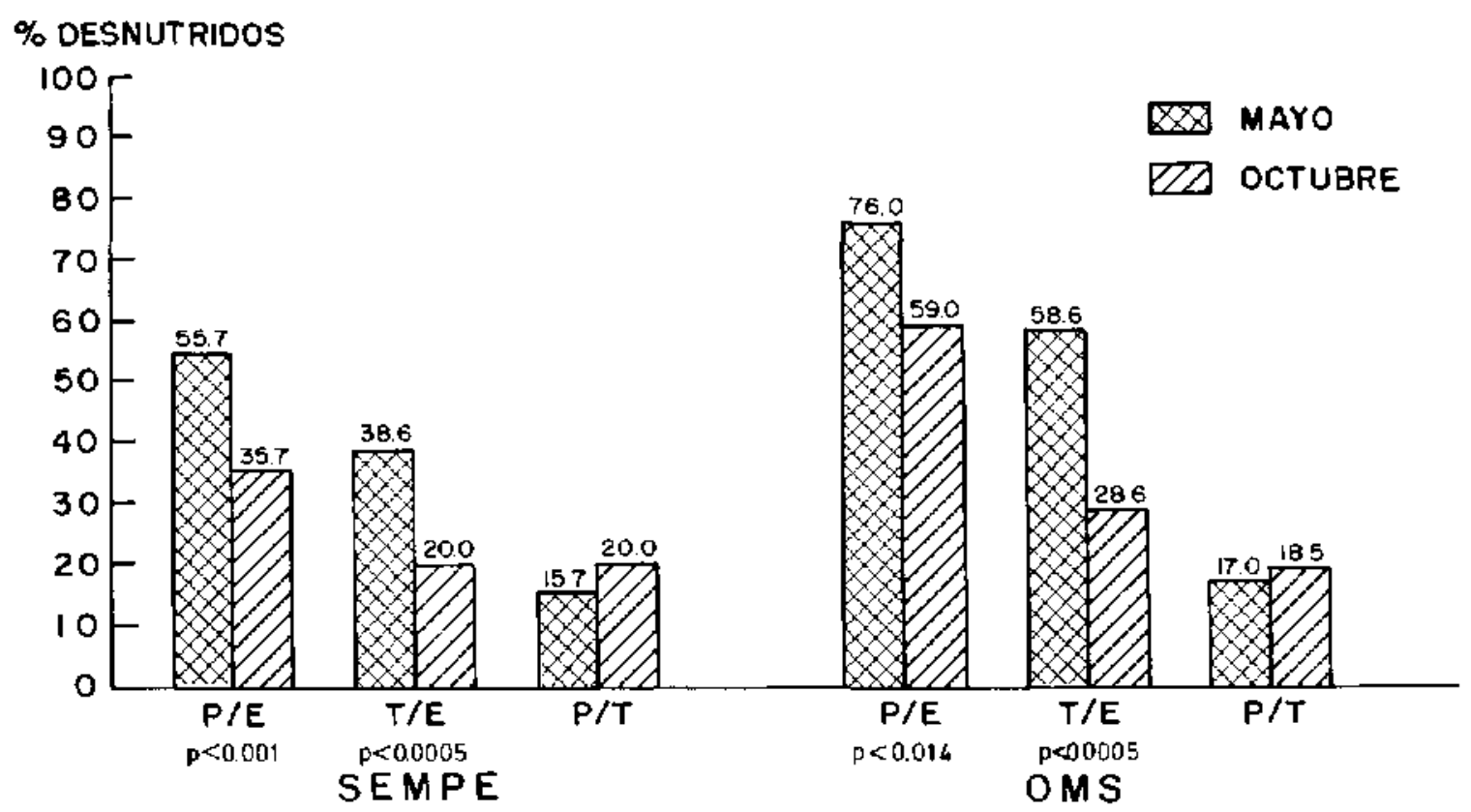

Figura 1. Variación del estado nutricional de 70 lactantes y precscolares asistentes a Centros de Recuperación Nutricional entre Mayo-Octubre 1984, según SEMPL y OMS. Comuna de La Florida. Santiago.

cercanas, ha recibido atención prioritaria en el pais gracias a los programas preventivos y de recuperación de la Salud. En el aspecto preventivo, ha jugado un rol fundamental et Programa Nacional de Nlimentación Complementaria (PNAC), que además de mejorar los hábitos alimentarios de lá población, está destinado a los sectores de menores ingresos mejorando el ingreso familiar entre un 4 y $35 \% 1{ }^{\circ}$, mejora el estado nutricional de los beneficiarios, aumenta el peso al nacer de los niños y la sobrevida en el primer año de vida ${ }^{11}$. Por último, cono para retirar los alimentos es obligatorio el control de salud, el PN $\Lambda C$ ha contribuido a elevar la cobertura $y$ frecuencia de estos controles contribuyendo a una detección precoz de las enfermedades.

En cuanto a tecuperación, existen los centros cerrados de recuperación mutricional de CONIN cuyo objetiwo es hacer desaparecer la desnutrición grave (Grado 2 y 3), que aún cuando afecta a un grupo escaso de niños, son de altísimo riesgo y tienen una alta incidencia en los costos de los servicios asjstenciales de salud. El éxito de este programa a nivel nacional es imegable, siendo la mortalidad de estos niños en los Centros de aperias an $3 \%$ egresando un $73 \%$ totalmente recuperado. De estos, un $78 \%$ continúan cum. pliendo su programa de control y mantienen un un estado nutritivo normal ${ }^{12}$. En el mismo tiempo se ha implementudo una antigua idea para recuperar a los desnutridos graves que es la colocación familiar (Colocación Familiar de Niños Desnutridos Severos - COFADE), con exce. lentes resultados en el Servicio de Salud Metropo. litano Sur Oriente ${ }^{13}$.

A pesar de que los desnutridos leves son citados con mayor frecuencia a controles de salud efectuados por mutricionistas y se les dá un aporte alimentario adicional, no existía un programa integral asistencial. nutricional, educacional y participativo que permitiese su rápida recuperación. En este sentido, la implementación de estos Centros Comuntarios Educativos Nutricionales, con participación de la propia comunidad interesada en resolver su problema y wo una asistencia técnica adecuada, es un avance frente a un problena que persiste a pesar de la eficiencia de otros programas.

La evaluación efectuada en este trabajo demuestra que si la intervención es efectuada con criterio técnico, se observar resultados en un plazo de 6 meses. En priner lugar, se requiere la buena asistencia de los niños; el grupo estudiado la tenia y de ahi sus resultados positivos. En segundo lugar, el aspecto nutricional debe ser cuidadosamente supervisado para que la dieta tenga las calorias necesarias que permitan la recuperación del niño, y debe existir preocupación especial. principalmente en lactantes, por la ingesta real del alimento entregado a los niños. En este sentido, seria recomendable que estas instituciones hicieran un esfuerzo por aumentar el aporte calórico para llegar a un mínimo de un $80 \%$ de los requerimientos según $\mathrm{FAO} / \mathrm{OMS} / 73^{5}$. En tercer lugar, debe hacerse una evaluación mensual del estado nutricional de los niños con patrones SEMPE y OMS, y además, estudiarse los incrementos de peso mensuales de cada niño para 
controlar su adecuada recuperación.

Por último, es fundamental integrar todas estas acciones con un programa educativo de salud y nutrición hacia la comunidad y personal de las instifuciones tendiente a mejorar en forma progresiva y permanente los factores que condicionan el estado nutricional del niño.

En nuestra evaluación nutricional encontramos una mejoría global de los indicadores pesof edud y talla/edad con los patrones SEMPE $y$ OMS que fueron estadisticamente significativos. En cambio, con el indicador peso/talla no hubo variación en los 6 meses observados, lo que puede atribuirse a la gran mejoría de la talla en los niños por la intervención nutricional.

Sin embargo, el aporte fundamental de estos Centros es lograr la participación de la comunidad en la toma de conciencia y solución del problema nutricional, que a pesar de los esfuerzos mancomurados del sector público y privado, aún persiste en nuestro medio, especialmente en los sectores poblaciorales de más bajos recursos.

\section{RESUMEN}

En el proyecto "Una acción integral para la prevención y tratamiento de la Desnutrición" se crearon 4 Centros Nutricionales para lactantes y preescolares desnutridos o en riesgo. El programa consistió en atención de 8 horas con estimulación psicomotora y dieta de $1.200 \mathrm{Kcal} / \mathrm{d}$ a. La craluación inicial en 70 nin̄os demostró que la dieta contenia $985 \mathrm{~K} \mathrm{cal}$; dia y la ingesta era de un $60 \%$ de ella. El estado nutricional peso/edad SEMPE demostraba $55.7 \%$ de desnutrición y OMS un $76 \%$. Talla/edad SEMPE tenía un déficit de $38.6 \%$ y OMS $58.6 \%$. Después de 6 meses de intervención nutricional, asistencial y educativa. la diela era de $1.123 \mathrm{Kcal} / \mathrm{dia}$, con una ingesta promedio de $75 \%$. La desnutrición peso/edad SEMPE habia disminuido a un $35.7 \%$ $(p<0.001)$ y OMS a un $59 \%(p<0.0014)$. Para talla/edad SEMPI la disminución fue al $20 \%$ $(\mathrm{p}<0.0005)$ y OMS a $28.6 \%(\mathrm{p}<0.0005)$. Esta recuperación nutricional demuestra que la evaluación cuidadosa de los Centros es fundamental para que se cumplan sus objetivos.

\section{AGRADECIMIENTOS}

Agradecemos expecialmente la colaboración y facilidades prestadas por las autoridades y personal de Salud del Servieiu Metropolitano de Sulud Sur Oriente de Santiago $y$ por las autoridades y personal del A tea de Desarrollo Social de la llustre Municipalidad de La Floridi.

\section{REFERENCIAS}

1. Raeznsfy, D., Oyarzo, C. Evolución teciente de la tasa de mortatiad iniantil. Rev. Méd. Chile 110: $784,1982$.

2. Scrra. I. Cerda, L.: Intento de evaluación del Programa de Alimentación Complementaria. Cuad. Med. Soc. 25: 30, 1984.

3. Ministerio de Sahd. Instituto Nacional de Fistadistica. Anuario 1982. Atenciones y Recursos. Santiago, Chile, 1983.

4. Unialad de Salud Familiar, INTA: Segundo Informs de Avance Proyecto IAF/INГC Diagrtóstico de la situación de salud. Conuna de La Plorida. Noviombre 1982 .

5. FAOIOMS/73. Necesidades de Encrgía y Proteinas. Rotma, FAO/Roma, 1973 (tAO Reuniones sobre mutricion No 52 OMS. Serie de Intomes Técnicos No $\leq 22)$.

6. Oliwers. S., Andrade. $M$, Dutur, E., Abala, C., Fio, F., Valiente, S.: Commusity participation in an educational program for preventing undernutition. Accepted XIII international Congress of Vutritios. Brighton, 1985.

7. SEMPEM.: Surveillance de la croissence de lenfant. References practiquas. Le Concours Medicale Suplemento No $43,1964$.

8. OMS-OPS: Medición del efecto nutricional de Programas de Alimentación Suplementaria a grupos vulnerables: WEO/TAP/79.1.

9. Puffer, R.. Serrano $C$. Caracteristicas de la mortalidad en la ninez. Otganización Panimericana de la Salud. Publicación Cicutíljea No 262. Washington. [) C. 1973

10. González, $N^{\prime}$, Infante, $A$, Mardones, $F_{2}$ Análisis del impacto de la atención primaria de saluđ sobre los indjeadores de salud y nutrición. Chile 1969.78. Pediatría. (Stgo.) 23: 192, 1980.

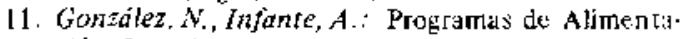
ción Complementaria del sector Salud en Chile. Bol. Of. Sanit. Panam. 89: $\$ 63,1980$.

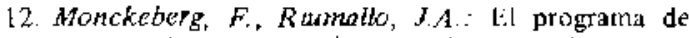
centros de Iccuperación rutricional en Chile: unat experiencia con la marginalidad. Fin CLPAL UNICEF. Pobreza Crítica en la niñez. A. Latina y el Caribe. F. Galotté, compilador, 1981.

13. Puentes, R., Escudero, P., Solari, M. et at Colocación Familiar de niños desnutridos scyeros (COFADE. Rev. Chil. Nuts. 12:133, 1984. 